\title{
Rayleigh waves in air saturated axisymmetrical soft porous media
}

\author{
J. F. Allard ${ }^{1, a)}$ O. Dazel, ${ }^{1}$ J. Descheemaeker, ${ }^{2}$ N. Geebelen, ${ }^{2}$ L. Boeckx ${ }^{2}$ and \\ W. Lauriks ${ }^{2}$ \\ ${ }^{1}$ Laboratoire d'Acoustique, Université du Maine, UMR CNRS 6613, Avenue Olivier Messiaen, \\ 72000 Le Mans, France \\ ${ }^{2}$ Laboratorium voor Akoestiek en Thermische Fysica, K. U. Leuven, Celestijnenlaan 200D, \\ 3001 Heverlee, Belgium
}

(Received 12 February 2009; accepted 15 May 2009; published online 9 July 2009)

\begin{abstract}
Bulk waves in axisymmetrical poroelastic layers having a given slowness in a plane perpendicular to the symmetry axis of the medium have been previously studied. The previous works are generalized to any angle between the plane where the slowness is defined and the symmetry axis. This allows a description of the Rayleigh waves created by a line source on free surfaces which are not perpendicular to the symmetry axis. An illustration is provided with measurements on a glass wool of the speed of the Rayleigh wave performed in three perpendicular directions and the evaluation of the rigidity parameters of the fibrous medium. (C) 2009 American Institute of Physics. [DOI: 10.1063/1.3159008]
\end{abstract}

\section{INTRODUCTION}

Fibrous media (glass wools, rock wools) and reticulated foams used for sound absorption or for damping are generally anisotropic. This anisotropy is generally neglected for the foams. Precise measurements show that the anisotropy can be very complex, but the simple axisymmetric model can provide an improved description of the mechanical properties of foams and fibrous media. ${ }^{1,2}$ Theoretical descriptions of the Biot waves in axisymmetric porous layers with a given slowness at the surface have been performed previously. ${ }^{3-5}$ The description is performed for layers with a symmetry axis perpendicular to this surface. Among these works, in Ref. 5 the recent $\left\{u^{s}, u^{t}\right\}$ formulation ${ }^{6}$ of the Biot theory is used, and a description of the bulk waves created by an excitation with a given wave number at the surface of the layer which is perpendicular to the axis of symmetry is given with the new formalism. A more general model is proposed in this work; any angle between the axis $Z$ normal to the surface and the symmetry axis $Z^{\prime}$ is allowed. A remaining problem for a prediction of the mechanical and acoustical properties of the axisymmetric porous media is the measurement of a complete set of dynamic rigidity parameters. Measurements are generally static or are performed at very low frequencies. ${ }^{1,2,7-11}$ For isotropic porous layers saturated by a light fluid, the Rayleigh wave is the one surface wave that can be observed. ${ }^{12}$ For isotropic soft structures, the speed of the Rayleigh wave can provide an estimation the shear modulus. ${ }^{13}$ With the general model, the speed and the damping of the Rayleigh wave created by a line source are predicted for different angles between $Z^{\prime}$ and $Z$ and different directions of the line source on the free surface where the Rayleigh wave propagates. As an illustration, the rigidity coefficients of a glass wool are evaluated at audible frequencies from measurements of the speed of the Rayleigh wave in three perpendicular directions. In Sec. II, the main results of

${ }^{a)}$ Electronic mail: jean-francois.allard@univ-lemans.fr. the work previously performed with the new formulation are recalled. The generalization is performed in Sec. III for any angle between $Z$ and $Z^{\prime}$. The prediction of the slowness of the Rayleigh waves is performed in Sec. IV. The evaluation of the rigidity parameters of a glass wool from Rayleigh wave speed measurements is performed in Sec. V.

\section{BIOT WAVES IN AN AXISYMMETRICAL LAYER WITH THE SYMMETRY AXIS NORMAL TO THE SURFACE}

\section{A. $\left\{\boldsymbol{u}^{s}, \boldsymbol{u}^{\dagger}\right\}$ representation}

Let $Z^{\prime}$ be the axis of symmetry of the transversely isotropic porous medium. In a first step the $Z$ axis normal to the free surface of the layer is parallel to $Z^{\prime}$. Biot ${ }^{14}$ extended the description of isotropic poroelastic media to transversely isotropic media. This description is slightly modified by taking into account the anisotropy of the acoustical parameters. Let $\boldsymbol{u}^{s}$ be the frame displacement and $\boldsymbol{u}^{f}$ be the air displacement. Let $\phi$ be the porosity. The total displacement $\boldsymbol{u}^{t}$ is defined by $\boldsymbol{u}^{t}=(1-\phi) \boldsymbol{u}^{s}+\phi \boldsymbol{u}^{f}$. Let $\sigma_{i j}^{t}$ be the total stress tensor of the frame. The strain-stress relations can be written under the hypothesis that the medium the frame is made of is much less compressible than air,

$$
-p=\tilde{K}_{\mathrm{eq}} \nabla \cdot \boldsymbol{u}^{t}, \quad \sigma_{i j}^{t}=\hat{\sigma}_{i j}-p \delta_{i j},
$$

where $p$ is the interstitial pressure, $\widetilde{K}_{\text {eq }}$ is the bulk modulus of the fluid modified by the thermal exchanges with the frame, ${ }^{15}$ and $\hat{\sigma}_{i j}$ is the in vacuo stress tensor of the frame which only depends on $\boldsymbol{u}^{s}$. Equations (1) show the simple dependence of $p$ on $\boldsymbol{u}^{t}$ and the contributions of the in vacuo stress tensor and of the pressure to the total frame stress tensor. Let $\varepsilon_{i j}$ be the strain tensor components of the frame. If $Z$ is parallel to the symmetry axis, the in vacuo stress-strain relations can be written as

$$
\hat{\sigma}_{x x}=(2 N+A) \varepsilon_{x x}+A \varepsilon_{y y}+F \varepsilon_{z z},
$$




$$
\begin{aligned}
& \hat{\sigma}_{y y}=A \varepsilon_{x x}+(2 N+A) \varepsilon_{y y}+F \varepsilon_{z z}, \\
& \hat{\sigma}_{z z}=F \varepsilon_{x x}+F \varepsilon_{y y}+C \varepsilon_{z z}, \\
& \hat{\sigma}_{y z}=2 L \varepsilon_{y z} \\
& \hat{\sigma}_{x z}=2 L \varepsilon_{x z} \\
& \hat{\sigma}_{x y}=2 N \varepsilon_{x y}
\end{aligned}
$$

where $A, F, N, C$, and $L$ are the rigidity parameters of the frame in vacuo. The viscoinertial interaction between the frame and the saturating fluid is described by a dynamic tortuosity $\widetilde{\alpha}_{z}(\omega)$ in the direction $Z$ and a dynamic tortuosity $\tilde{\alpha}_{x}(\omega)$ for all directions perpendicular to the symmetry axis. The dynamic tortuosity is related to the effective density $\tilde{\rho}_{\text {ef }}$ defined in Ref. 15 by $\widetilde{\alpha}=\tilde{\rho}_{\text {ef }} / \rho_{0}$, where $\rho_{0}$ is the density of air.

\section{B. Bulk waves in the layer}

Let $\boldsymbol{q}$ be the slowness vector of a plane wave. A set of axes $X, Y, Z$, where the $y$ component $q_{y}=0$ is used. The plane $X, Z$ is a meridian plane parallel to the direction of propagation. In Ref. 5 the displacement components are written as

$$
\begin{aligned}
& u_{x}^{s}=a_{x} \exp \left[i \omega\left(t-q_{x} x-q_{z} z\right)\right], \\
& u_{y}^{s}=a_{y} \exp \left[i \omega\left(t-q_{x} x-q_{z} z\right)\right], \\
& u_{z}^{s}=a_{z} \exp \left[i \omega\left(t-q_{x} x-q_{z} z\right)\right], \\
& u_{x}^{t}=b_{x} \exp \left[i \omega\left(t-q_{x} x-q_{x} z\right)\right], \\
& u_{y}^{t}=b_{y} \exp \left[i \omega\left(t-q_{x} x-q_{x} z\right)\right], \\
& u_{z}^{t}=b_{z} \exp \left[i \omega\left(t-q_{x} x-q_{z} z\right)\right] .
\end{aligned}
$$

The wave equations projected on the axes lead to a system of six equations which relate $a_{x}, a_{y}, a_{z}, b_{x}, b_{y}, b_{z}$. This system splits into two subsystems. A system of four equations relates the displacement components of the waves polarized in the meridian plane with $a_{y}=b_{y}=0$. It can be written as

$$
\begin{aligned}
& V_{i, 1} a_{x}+V_{i, 2} a_{z}+V_{i, 3} b_{x}+V_{i, 4} b_{z}=0, \\
& i=1,2,3,4 .
\end{aligned}
$$

The coefficients $V_{i, j}$ are given in Eq. (13) of Ref. 5. The determinant of this system is equal to 0 , leading to the following equation:

$$
T_{3} q_{z}^{6}+T_{2} q_{z}^{4}+T_{1} q_{z}^{2}+T_{0}=0 .
$$

The coefficients $T_{i}$ are polynomials of degree $3-i$ in $q_{x}^{2}$,

$$
\begin{aligned}
& T_{0}=T_{0,6} q_{x}^{6}+T_{0,4} q_{x}^{4}+T_{0,2} q_{x}^{2}+T_{0,0}, \\
& T_{1}=T_{1,4} q_{x}^{4}+T_{1,2} q_{x}^{2}+T_{1,0},
\end{aligned}
$$

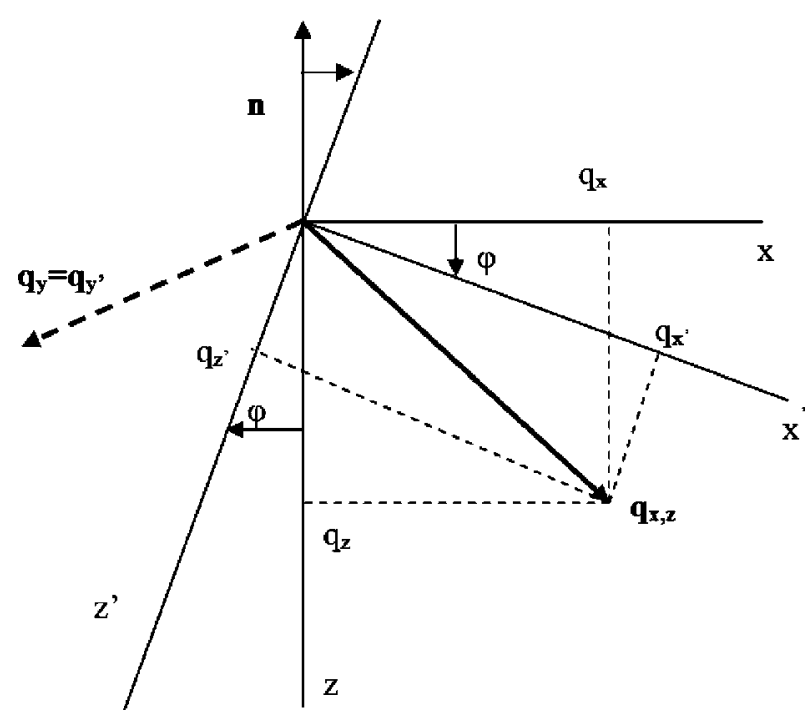

FIG. 1. The components of the slowness vector $\mathbf{q}$ in the $O x z$ plane.

$$
T_{2}=T_{2,2} q_{x}^{2}+T_{2,0}
$$

The coefficients $T_{3}$ and $T_{i, j}$ are expressed in Eqs. (15)-(27) of Ref. 5. The components $b_{x}, a_{x}$, and $a_{z}$ can be expressed as a function of $b_{z}$ using three equations of the system (14). They are given in Eqs. (29)-(31) of Ref. 5 for a normalization factor $n=b_{z}$.

For the waves polarized in the direction perpendicular to the meridian plane the $z$ wave number components satisfies the following equation:

$$
\begin{aligned}
& q_{z}^{2}+T_{0}^{\prime}=0, \\
& T_{0}^{\prime}=T_{0,2}^{\prime} q_{x}^{2}+T_{0,0}^{\prime},
\end{aligned}
$$

where $T_{0,2}^{\prime}$ and $T_{0,0}^{\prime}$ can be obtained from Eq. (11) of Ref. 5. The component $b_{y}$ is expressed in Eq. (11) of Ref. 5 as a function of the new normalization factor $n=a_{y}$.

\section{SYMMETRY AXIS DIFFERENT FROM THE NORMAL TO THE SURFACE}

\section{A. Geometry and wave number components}

Let $Z$ be the axis normal to the free surface of the layer, $Z^{\prime}$ the axis of symmetry of the medium, and without any loss of generality, $X$ the axis on the surface of the layer in the plane $Z, Z^{\prime}$. The slowness vector $\boldsymbol{q}$ of a wave propagating in the poroelastic medium has three components $q_{x}, q_{y}, q_{z}$ in the set $X, Y, Z$ and three components $q_{x^{\prime}}, q_{y}, q_{z^{\prime}}$ in the set $X^{\prime}, Y^{\prime}, Z^{\prime}$. The description of the different bulk waves with slowness components $q_{x}, q_{y}$ on the surface is more difficult than in Sec. II because these components are defined in the set $X, Y, Z$ but in Eqs. (1)-(7) $x, y$, and $z$ must be replaced by $x^{\prime}, y^{\prime}$, and $z^{\prime}$, and Eqs. (14)-(18) which relate parameters in the $X, Y, Z$ set must be modified. The components $q_{x}, q_{x^{\prime}}, q_{z}, q_{z^{\prime}}$ are represented in Fig. 1 . The vector $\boldsymbol{q}_{x, y}$ is the slowness vector in the $X, Z$ plane. The angle between $Z$ and $Z^{\prime}$ is $\varphi$. In Fig. $1,0>\varphi>-\pi / 2$. The $q$ components in both systems are related by

$$
q_{x^{\prime}}=q_{x} \cos \varphi-q_{z} \sin \varphi
$$




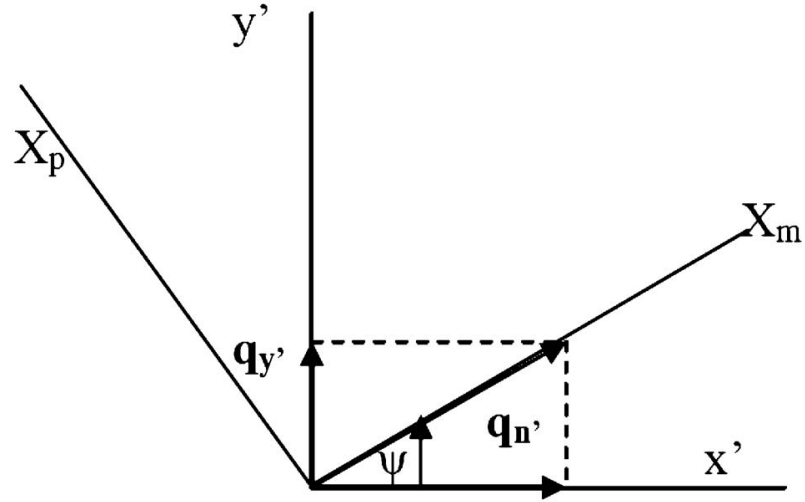

FIG. 2. The $O x^{\prime} y^{\prime}$ plane.

$$
\begin{aligned}
& q_{y^{\prime}}=q_{y}, \\
& q_{z^{\prime}}=q_{z} \cos \varphi+q_{x} \sin \varphi .
\end{aligned}
$$

Using $\bar{q}_{x}=q_{x} / \cos \varphi$ instead of $q_{x}, q_{x^{\prime}}$ is given by

$$
q_{x^{\prime}}=\bar{q}_{x}-q_{z^{\prime}} \tan \varphi \text {. }
$$

The components $q_{x^{\prime}}$ and $q_{y^{\prime}}$ define with the axis $Z^{\prime}$ the meridian plane parallel to the slowness vector. Let $\boldsymbol{q}_{n^{\prime}}$ be the vector defined by

$$
\boldsymbol{q}_{n^{\prime}}=\mathbf{e}_{x^{\prime}} q_{x^{\prime}}+\mathbf{e}_{y} q_{y}
$$

where $\mathbf{e}_{x^{\prime}}$ and $\mathbf{e}_{y}$ are the unit vectors in the directions $X^{\prime}$ and $Y$, respectively. The components $q_{n^{\prime}}$ and $q_{z^{\prime}}$ are related by the same relation as $q_{x}$ and $q_{z}$ in Sec. II. The squared modulus $q_{n^{\prime}}^{2}$ can be written as

$$
\begin{aligned}
& q_{n^{\prime}}^{2}=\bar{q}_{n}^{2}+H q_{z^{\prime}}+I q_{z^{\prime}}^{2}, \\
& \bar{q}_{n}^{2}=q_{y}^{2}+\bar{q}_{x}^{2}, \\
& H=-2 \bar{q}_{x} \tan \varphi, \\
& I=\tan ^{2} \varphi .
\end{aligned}
$$

\section{B. Waves polarized in a meridian plane}

Equation (15) can be used to predict the slowness components $q_{z}$ but $q_{x}^{2}$ in the expressions of the coefficients $T_{1}, T_{2}, T_{3}$ must be replaced by $q_{n^{\prime}}^{2}$ which is expressed in Eq. (24) as a polynomial in $q_{z^{\prime}}$. The slowness components $q_{z^{\prime}}$ satisfy a new polynomial expression,

$$
A_{6} q_{z^{\prime}}^{6}+A_{5} q_{z^{\prime}}^{5}+A_{4} q_{z^{\prime}}^{4}+A_{3} q_{z^{\prime}}^{3}+A_{2} q_{z^{\prime}}^{2}+A_{1} q_{z^{\prime}}+A_{0}=0 .
$$

The polynom is no longer a cubic in $q_{z^{\prime}}^{2}$ as in Eq. (15). The coefficients $A_{i}$ are given in Appendix. For each root, $q_{x^{\prime}}$ can be evaluated from Eq. (22). The meridian plane parallel to the slowness vector is defined by the direction $Z^{\prime}$, and in the plane perpendicular to $Z^{\prime}$ by the direction of the vector $\boldsymbol{q}_{n^{\prime}}$ $=\boldsymbol{q}_{x^{\prime}}+\boldsymbol{q}_{y^{\prime}}$, where $\boldsymbol{q}_{y^{\prime}}=\boldsymbol{q}_{y}$ (see Fig. 2). This direction is denoted as $X_{m}$ and the orthogonal direction in the same plane is denoted as $X_{p}$. Equations (29)-(31) of Ref. 5 can be used to evaluate the coefficients $a_{m}, a_{z^{\prime}}, b_{m}$ for a given $n=b_{z^{\prime}}$.

\section{Waves polarized in the direction perpendicular to a meridian plane}

The equations of motion projected in $X_{p}$ direction can be written as

$$
A_{2}^{\prime} q_{z^{\prime}}^{2}+A_{1}^{\prime} q_{z^{\prime}}+A_{0}^{\prime}=0,
$$

and the same relation relates $b_{p}$ and $a_{p}$, and $b_{y}$ and $a_{y}$ in Sec. II. The coefficients $A_{i}^{\prime}$ are given in Appendix. For each root $q_{z^{\prime}}, q_{x^{\prime}}$ can be evaluated from Eq. (22). The chosen normalization factor $n$ for this wave is $n=a_{p}$.

\section{Symmetry axis in the interface plane}

If $\varphi=\pi / 2$, the symmetry axis $Z^{\prime}$ is in the interface plane. Equations (19)-(21) cannot be used. The direction $X$ at the surface of the layer can be chosen parallel to $Z^{\prime}$, and $q_{z^{\prime}}$ $=q_{x}$ is known. The unknown quantity is $\boldsymbol{q}_{n^{\prime}}=\boldsymbol{q}_{y}+\boldsymbol{q}_{x^{\prime}}$. The previous Eq. (28) is replaced by

$$
\begin{aligned}
& S_{0} q_{n^{\prime}}^{6}+S_{1} q_{n^{\prime}}^{4}+S_{2} q_{n^{\prime}}^{2}+S_{3}=0, \\
& S_{0}=T_{0,6}, \\
& S_{1}=T_{0,4}+T_{1,4} q_{x}^{2}, \\
& S_{2}=T_{0,2}+T_{1,2} q_{x}^{2}+T_{2,2} q_{x}^{4}, \\
& S_{3}=T_{0,0}+T_{1,0} q_{x}^{2}+T_{2,0} q_{x}^{4}+T_{3} .
\end{aligned}
$$

For the waves polarized perpendicularly to the meridian plane, Eq. (29) is replaced by

$$
T_{0,2}^{\prime} q_{n^{\prime}}^{2}+T_{0,0}^{\prime}+q_{x}^{2}=0 .
$$

\section{E. Stress tensor components and velocities in the $x y z$ axis set}

For each wave, let $\psi$ be the angle between the direction $X^{\prime}$ and $X_{m}$ given by

$$
\begin{aligned}
& \cos \psi=\frac{q_{x^{\prime}}}{\left(q_{x^{\prime}}^{2}+q_{y^{\prime}}^{2}\right)^{1 / 2}}, \\
& \sin \psi=\frac{q_{y^{\prime}}}{\left(q_{x^{\prime}}^{2}+q_{y^{\prime}}^{2}\right)^{1 / 2}} .
\end{aligned}
$$

(See Fig. 2). For each wave the displacement components and the slowness components in the $X^{\prime}, Y^{\prime}, Z^{\prime}$ are obtained by a rotation around $Z^{\prime}$ of angle $\psi$. A second rotation performed around $Y$ gives the same components in the set $X, Y, Z$. The stress components in the $X^{\prime}, Y^{\prime}, Z^{\prime}$ set and the pressure can be calculated with Eqs. (1)-(7), where $x, y, z$ are replaced by $x^{\prime}, y^{\prime}, z^{\prime}$. The stress components of the frame in vacuo in the $X, Y, Z$ set are given by

$$
\hat{\sigma}_{z z}=\hat{\sigma}_{x^{\prime} x^{\prime}} \sin ^{2} \varphi-2 \hat{\sigma}_{x^{\prime} z^{\prime}} \sin \varphi \cos \varphi+\hat{\sigma}_{z^{\prime} z^{\prime}} \cos ^{2} \varphi,
$$




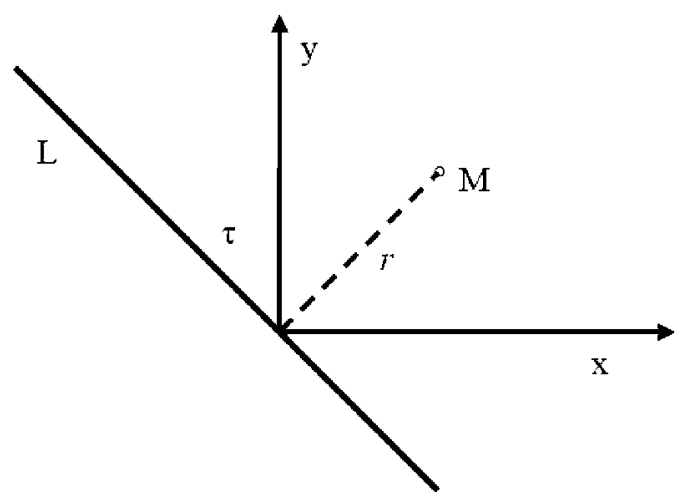

FIG. 3. The line source $L$ on the free surface.

$$
\begin{aligned}
\hat{\sigma}_{z x}= & -\hat{\sigma}_{x^{\prime} x^{\prime}} \sin \varphi \cos \varphi+\hat{\sigma}_{z^{\prime} x^{\prime}}\left(\cos ^{2} \varphi-\sin ^{2} \varphi\right) \\
& +\hat{\sigma}_{z^{\prime} z^{\prime}} \sin \varphi \cos \varphi, \\
\hat{\sigma}_{z y}= & -\hat{\sigma}_{x^{\prime} z^{\prime}} \sin \varphi+\hat{\sigma}_{z^{\prime} y^{\prime}} \cos \varphi .
\end{aligned}
$$

\section{RAYLEIGH WAVE CREATED BY A LINE SOURCE}

\section{A. Normal stress line source}

A simplified model is used. The line source of thickness $2 a$ is located around an axis $\mathbf{L}$. The source creates at the surface of the layer a stress $\sigma_{z z}$ applied to the frame which is given by

$$
\sigma_{z z}(r, t)=g(r) h(t)
$$

where $r$ is the distance from $\mathbf{L}$ (see Fig. 3). Using the spatial and the time Fourier transforms $\widetilde{g}(q)$ and $\tilde{h}(\omega)$ of $g(r)$ and $h(t)$, this excitation can be replaced in the frequency domain by a superposition of excitations with a space-time dependence exp $i \omega\left(t-i q_{r} r\right)$. The spatial Fourier transform and the inverse transformation can be written as

$$
\begin{aligned}
& \tilde{g}\left(q_{r}\right)=\int_{-a}^{a} g(r) \exp \left(i \omega q_{r} r\right) d r \\
& g(r)=\frac{1}{2 \pi} \int_{-\infty}^{\infty} \tilde{g}\left(q_{r}\right) \exp \left(-i \omega q_{r} r\right) \omega d q_{r},
\end{aligned}
$$

and $\sigma_{z z}(r, t)$ can be rewritten as

$$
\begin{aligned}
\sigma_{z z}(r, t)= & \frac{1}{4 \pi^{2}} \int_{-\infty}^{\infty} \int_{-\infty}^{\infty} \tilde{h}(\omega) \widetilde{g}\left(q_{r}\right) \exp ( \\
& \left.-i \omega q_{r} r\right) \exp (i \omega t) \omega d t d q_{r} .
\end{aligned}
$$

Let $\tau$ be the angle between $\mathbf{L}$ and the direction $Y$. Let $M$ be the location of the receiver. The product $q_{r} r$ can be replaced by $q_{x} x+q_{y} y$, where $q_{x}=q_{r} \cos \tau, q_{y}=q_{r} \sin \tau$. The vertical displacement $U_{z}(x, y, t)$ of the frame surface at $M$ of coordinates $x$ and $y$ is a superposition of displacements $\widetilde{u}_{z}^{s}$ related to the elementary excitations $\widetilde{\sigma}_{z z}=\exp \left[-i \omega\left(q_{x} x+q_{y} y\right)\right] \exp (i \omega t)$,

$$
\begin{aligned}
U_{z}(x, y, t)= & \frac{1}{4 \pi^{2}} \int_{-\infty}^{\infty} \int_{-\infty}^{\infty} \tilde{h}(\omega) \tilde{u}_{z}^{s}\left(q_{x}, q_{y}, \omega\right) \\
& \times \exp \left(-i \omega q_{r} r\right) \exp (i \omega t) \omega d t d q_{r} .
\end{aligned}
$$

The calculation of $\widetilde{u}_{z}^{s}\left(q_{x}, q_{y}, \omega\right)$, which depends on $\tau, q_{r}$, and $\omega$, is described in Sec. IV B.

\section{B. Vertical displacement created by an elementary excitation}

\section{Semi-infinite layers, $Z$ and $Z^{\prime}$ nonparallel}

For a given set $\left(q_{x}, q_{y}\right)$ there are three waves polarized in their meridian plane and one wave polarized in the direction normal to the meridian plane which decreases when $z$ increases. For a wave numbered $k$, the pressure $p^{k}$, the stress components $\hat{\sigma}_{i j}^{k}$ of the frame in vacuo, and the displacement components $a_{3}^{k}$ and $b_{3}^{k}, k=1,4$, are calculated with a normalization factor $n_{k}=1$. Let $p$ and $v_{z}$ be the pressure and the $z$ component of the air velocity in the free air at the air-porous medium interface. Let $\theta$ be the complex angle between the wave number vector of the wave emitted by the porous layer in the free air and the normal $\boldsymbol{n}$ represented in Fig. 1. The spatial dependence of this wave is $\exp \left[-i \omega\left(q_{x} x+q_{y} y\right.\right.$ $\left.-q_{0} z \cos \theta\right)$, where $q_{0}$ is the slowness in the free air. There are two determinations of $\cos \theta$ which is given by

$$
\cos \theta= \pm \frac{\sqrt{q_{0}^{2}-q_{x}^{2}-q_{y}^{2}}}{q_{0}} .
$$

For the determination with $\operatorname{Im} \cos \theta<0$ the wave in air decreases when the distance from the interface increases. The ratio $p / v_{z}$ is given by

$$
p / v_{z}=-Z_{0} / \cos \theta
$$

where $Z_{0}$ is the characteristic impedance of air. The boundary conditions at the surface of the porous layer can be written as

$$
\sum_{k=1,2,3,4} n_{k} \hat{\sigma}_{z z}^{k}=1
$$

where the factor $\exp \left[i \omega\left(t-q_{x} x-q_{z} z\right)\right]$ is removed,

$$
\begin{aligned}
& \sum_{k=1,2,3,4} n_{k} \hat{\sigma}_{z x}^{k}=0, \\
& \sum_{k=1,2,3,4} n_{k} \hat{\sigma}_{z y}^{k}=0, \\
& j \omega Z_{0} \sum_{k=1,2,3,4} n_{k} b_{z}^{k}=-\sum_{k=1,2,3,4} n_{k} p^{k} \cos \theta,
\end{aligned}
$$

The contributions are obtained as indicated in Sec. IV A. The four coefficients $n_{k}$ are obtained from Eqs. (47)-(50) and the $z$ component of the frame displacement is given by

$$
\tilde{u}_{z}^{s}\left(q_{x}, q_{y}, \omega\right)=\sum_{k=1-4} n_{k} a_{3}^{k} .
$$

\section{Layer of finite thickness, $Z$ and $Z$ ' parallel}

The case of a layer of finite thickness with $Z$ and $Z^{\prime}$ nonparallel is beyond the scope of the work. For $Z$ and $Z^{\prime}$ 
TABLE I. Parameters for the glass wool. $F=A=0$.

\begin{tabular}{lc}
\hline \hline Flow resistivity $\sigma^{x^{\prime}}\left(\mathrm{N} \mathrm{m}^{-4} \mathrm{~s}\right)$ & 4000 \\
Flow resistivity $\sigma^{z^{\prime}}\left(\mathrm{N} \mathrm{m}^{-4} \mathrm{~s}\right)$ & 8000 \\
Porosity $\phi$ & 0.98 \\
Tortuosity $\alpha_{\infty}^{x^{\prime}}=\alpha_{\infty}^{z^{\prime}}$ & 1.1 \\
Thermal permeability $\mathrm{k}_{0}^{\prime}\left(\mathrm{m}^{2}\right)$ & $6 \times 10^{-9}$ \\
Viscous dimension $\Lambda^{x^{\prime}}(\mu \mathrm{m})$ & 200 \\
Viscous dimension $\Lambda^{z^{\prime}}(\mu \mathrm{m})$ & 140 \\
Thermal dimension $\Lambda^{\prime}(\mu \mathrm{m})$ & 500 \\
$N(\mathrm{kPa})$ & $260 \times(1-01 j)$ \\
$C(\mathrm{kPa})$ & $46 \times(1-01 j)$ \\
$L(\mathrm{kPa})$ & $125 \times(1-01 j)$ \\
Density $\rho_{s}\left(\mathrm{~kg} / \mathrm{m}^{3}\right)$ & 32 \\
\hline
\end{tabular}

parallel Eq. (49) must be removed. Equations (47), (48), and (50) are always valid but the summations must include the six waves polarized in the meridian plane. Let $l$ be the thickness of the layer. The supplementary boundary conditions for a layer bonded to a rigid impervious backing are

$$
\begin{aligned}
& \sum_{k=1-6} n_{k} \exp \left(-i \omega q_{z}^{k} l\right) b_{z}^{k}=0, \\
& \sum_{k=1-6} n_{k} \exp \left(-i \omega q_{z}^{k} l\right) a_{z}^{k}=0, \\
& \sum_{k=1-6} n_{k} \exp \left(-i \omega q_{z}^{k} l\right) a_{x}^{k}=0 .
\end{aligned}
$$

\section{Rayleigh pole and Rayleigh waves}

Some properties of Rayleigh poles and Rayleigh waves are shown for the porous medium characterized by the parameters given in Table $\mathrm{I}$. The Poisson ratios are equal to 0 and $F=A=0$. Let $q_{p}$ be the location of the Rayleigh pole in the complex $q_{r}$ plane. When $q_{r} \rightarrow q_{p}$ the vertical displacement $\widetilde{u}_{z}^{s} \rightarrow \infty$. An iterative method ${ }^{13}$ can be used to predict $q_{p}$. An approximate value of $q_{p}$ is used to start the iteration. Due to the large difference between the air density and the frame density, the displacement of the frame created by a mechanical excitation is not noticeably modified by air and in a first approximation the porous frame can be replaced by the elastic frame in vacuo. It has been shown by Royer and Dieulesaint $^{16}$ [see Eq. (5.61) of Ref. 16] that $q_{p}$ for an axisymmetric elastic medium is one of the roots of a polynomial for the three geometries represented in Fig. 4 where the axis of symmetry $Z^{\prime}$ is vertical. The axis normal to the free sur-

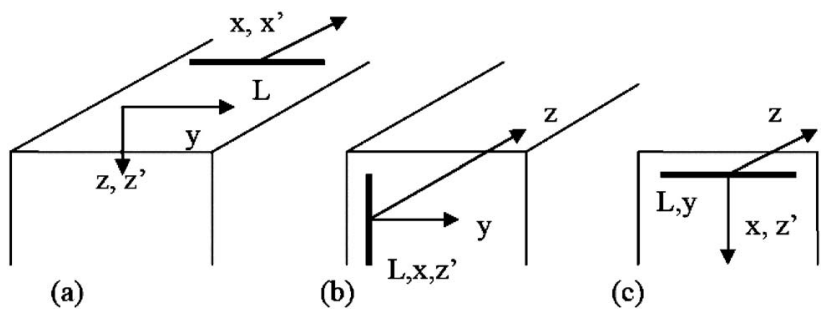

FIG. 4. Different geometries where the wave number in the free surface of the Rayleigh wave is a root of a simple polynomial expression. The axis of symmetry $Z^{\prime}$ is vertical. face is Z. In Fig. 4(a) the free surface where the Rayleigh wave propagates is a plane perpendicular to the axis of symmetry. In Figs. 4(b) and 4(c) the Rayleigh wave propagates in planes parallel to the axis of symmetry, in a direction perpendicular to the axis of symmetry in Fig. 4(b), and in a direction parallel to the axis of symmetry in Fig. 4(c). For the cases a, b, c, the polynomial can be written, respectively, as

$$
\begin{gathered}
L\left[(2 N+A)-q_{p}^{-2} \rho_{s}\right]\left(q_{p}^{-2} \rho_{s}\right)^{2}-C\left(L-q_{p}^{-2} \rho_{s}\right)[(2 N+A) \\
\left.-\frac{F^{2}}{C}-q_{p}^{-2} \rho_{s}\right]^{2}=0
\end{gathered}
$$

$$
\begin{aligned}
& N\left[(2 N+A)-q_{p}^{-2} \rho_{s}\right]\left(q_{p}^{-2} \rho_{s}\right)^{2}-(2 N+A)\left(N-q_{p}^{-2} \rho_{s}\right)[(2 N \\
& \left.+A)-\frac{A^{2}}{(2 N+A)}-q_{p}^{-2} \rho_{s}\right]^{2}=0 \\
& L\left(C-q_{p}^{-2} \rho_{s}\right)\left(q_{p}^{-2} \rho_{s}\right)^{2}-(2 N+A)\left(L-q_{p}^{-2} \rho_{s}\right)[C \\
& \left.\quad-\frac{F^{2}}{(2 N+A)}-q_{p}^{-2} \rho_{s}\right]^{2}=0
\end{aligned}
$$

where $\rho_{s}$ is the density of the frame. For the case a, b, c, in the absence of damping, it is shown in Ref. 16 that $q_{p}^{-2} \rho_{s}$ is located in the following intervals, respectively:

$$
\begin{aligned}
& 0<q_{p}^{-2} \rho_{s}<L, \quad(2 N+A)-\frac{F^{2}}{C}, \\
& 0<q_{p}^{-2} \rho_{s}<N, \quad(2 N+A)-\frac{A^{2}}{(2 N+A)}, \\
& 0<q_{p}^{-2} \rho_{s}<L, \quad C-\frac{F^{2}}{(2 N+A)} .
\end{aligned}
$$

The speed of the Rayleigh wave $1 / \operatorname{Re} q_{p}$ and the imaginary part of the slowness $\operatorname{Im} q_{p}$ calculated with the Biot model at 2 and $4 \mathrm{kHz}$ with the parameters of Table I are compared in Table II with the predictions obtained with Eqs. (55)-(57). The incompressibility $\tilde{K}_{\text {eq }}$ of the saturating air is predicted with the Lafarge model ${ }^{17}$ and the dynamic tortuosities $\widetilde{\alpha}^{i}$ in Eq. (3) of Ref. 5 are predicted with the model by Johnson et $a l .{ }^{18}$ For the geometry $\mathrm{b}$ the phase speed $1 / \operatorname{Re} q_{p}$ and $\operatorname{Im} q_{p}$ are shown in Figs. 5 and 6. The predictions with the Biot model for the speeds are very close to the ones obtained with Eqs. (58)-(60). Beyond $200 \mathrm{~Hz}$, the difference between the imaginary part of the slowness evaluated with Eq. (56) and the evaluation obtained with the Biot theory decreases when frequency increases because the viscous interaction decreases when frequency increases ${ }^{15}$ and the inertial forces become dominant. The same trends can be observed for the other geometries. A second property which has been previously noticed by Feng and Johnson ${ }^{12}$ for isotropic porous media saturated by a light fluid is the absence of contribution of other poles. This has been checked for the medium with the parameters of Table I by predicting the displacement nor- 
TABLE II. Phase speed $1 / \operatorname{Re} q_{p}$ and slowness imaginary part $\operatorname{Im} q_{p}$ for the Rayleigh pole in the geometries a, b, c of Fig. 4 and speed of the Rayleigh wave contribution.

\begin{tabular}{cccccccc}
\hline \hline & $\begin{array}{c}\text { Frame in vacuo } \\
\text { Speed }(\mathrm{m} / \mathrm{s})\end{array}$ & $\begin{array}{c}\text { Frame in vacuo } \\
\operatorname{Im} q_{p}\left(10^{-3} \mathrm{~s} / \mathrm{m}\right)\end{array}$ & $\begin{array}{c}\text { Biot } \\
\text { Speed }\end{array}$ & $\begin{array}{c}2 \mathrm{kHz} \\
\operatorname{Im} q_{p}\end{array}$ & $\begin{array}{c}\text { Biot } \\
\text { Speed }\end{array}$ & $\begin{array}{c}4 \mathrm{kHz} \\
\operatorname{Im} q_{p}\end{array}$ & $\begin{array}{c}\text { Rayleigh wave } \\
\text { Speed 3 kHz }\end{array}$ \\
\hline $\mathrm{a}$ & 51.00 & -0.978 & 50.65 & -1.12 & 50.72 & -1.07 & 50.3 \\
$\mathrm{~b}$ & 79.08 & -0.631 & 78.54 & -0.705 & 78.66 & -0.680 & 76.9 \\
$\mathrm{c}$ & 36.00 & -1.39 & 35.76 & -1.60 & 35.8 & -1.52 & 35.1 \\
\hline \hline
\end{tabular}

mal to the surface as a function of time for the three cases of Fig. 4. The signal $g(t)$ is a windowed sine at $3 \mathrm{kHz}$. The source is a line source with a space dependence $h(t)$ $=\cos b x,|x|<\pi /(2 b), h(t)=0,|x|>\pi /(2 b), b=\pi \times 4 \times 10^{3}$. The vertical velocity is obtained as a function of $t$ from Eq. (44) at distances of 3 and $5 \mathrm{~cm}$ from the line source. There is only one time interval where the velocity is noticeable and in this interval the velocity is approximately a replica of $h(t)$. The vertical velocity is represented for $r=3 \mathrm{~cm}$ in Fig. 7 for the case (a). The speed of the replica evaluated from the correlation function between both functions of time at $r$ $=3 \mathrm{~cm}$ and $r=5 \mathrm{~cm}$ is given in the last column of Table II. The speed is close to the predicted $1 / \operatorname{Re} q_{p}$ in all cases and this signal can be identified as the Rayleigh pole contribution. The specific properties of the Rayleigh waves for current isotropic or anisotropic air saturated porous sound absorbing media are related to the small rigidity and the large structural damping of these media. The order of magnitude of the wave speed is around $50 \mathrm{~m} / \mathrm{s}$ and the order of magnitude of the loss angle is 0.1. Due to the small wavelengths, it is possible to obtain at acoustical frequencies a signal which is mainly a Rayleigh wave contribution at a small distance from the source. This was previously predicted and observed for isotropic media. ${ }^{19}$ Another consequence is the weak modification of the Rayleigh wave contribution when the thickness of the layer decreases. For a layer of thickness $l=2 \mathrm{~cm}$ of the medium defined in Table I, the vertical displacement created by the line source was predicted at $r=3$ and $6 \mathrm{~cm}$ for the geometry a. The speed of the contribution is

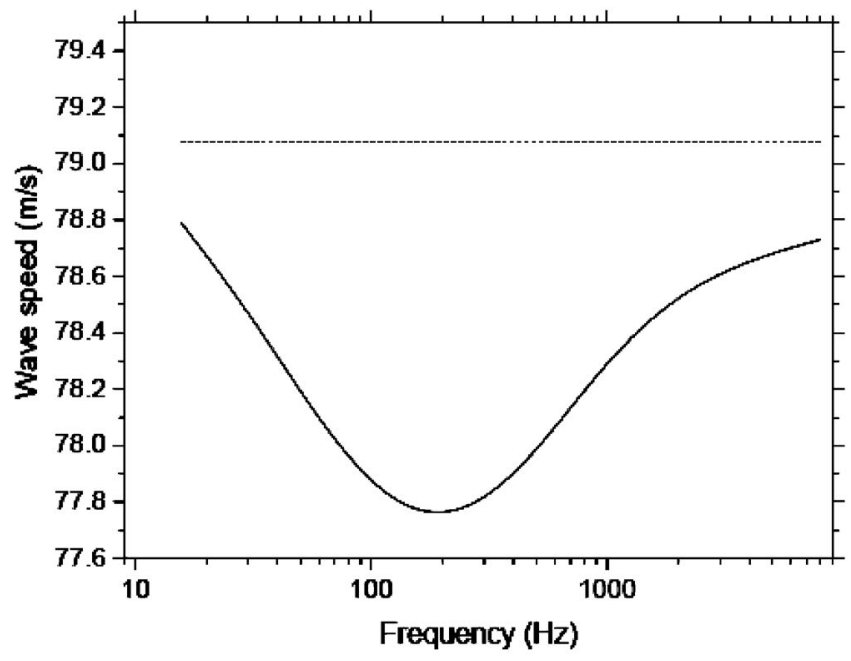

FIG. 5. Phase speed $1 / \operatorname{Re} q_{p}$ for the geometry b of Fig. 4 as a function of frequency; Biot (---); frame in vacuo (---). modified by less than $4 \%$ when the thickness decreases from infinity to $2 \mathrm{~cm}$.

\section{COMPARISONS BETWEEN MEASUREMENT AND PREDICTION OF RAYLEIGH WAVE SPEEDS AND EVALUATION OF RIGIDITY COEFFICIENTS}

The material is a layer of glass wool. A similar material was studied by Tarnow. ${ }^{2}$ The material is transversely isotropic and the axis of symmetry $Z^{\prime}$ is perpendicular to the large faces of the layer. The Poisson coefficients of fibrous media such as glass wool and rock wool can be neglected, and $F$ $=A=0$. It is shown in what follows that the set of rigidity parameters obtained from measurements of the speed of the Rayleigh wave contribution could be close to the one of Table I. The source field is created by a metal blade glued on the surface of the layer excited by a magnetic field which vibrates in the direction perpendicular to the surface. The time signal is a burst sine signal of ten periods. The frame velocity component perpendicular to the surface where the Rayleigh wave propagates is measured with a Polytec laser Doppler vibrometer. The experimental setup is represented in Fig. 8. The wave speed is evaluated from the measured times of flight related to different distances from the source for the three geometries of Fig. 4. The laser beam can be displaced along the surface using a movable mirror and a positioning system. Like in the simulations, only one replica appears in the velocity distributions, and it can be considered as a Rayleigh wave contribution. The contribution is localized at different distances from the line source from 3 to $6 \mathrm{~cm}$. The time of flight is determined using a cross correlation of the

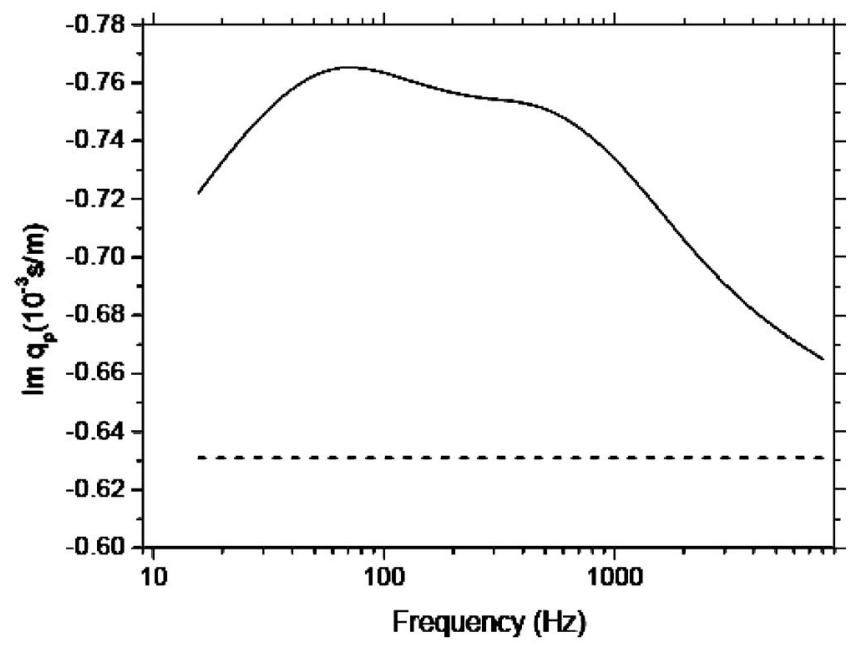

FIG. 6. Damping $\operatorname{Im} q_{p}$ for the geometry b of Fig. 4 as a function of frequency; Biot (---); frame in vacuo (---). 


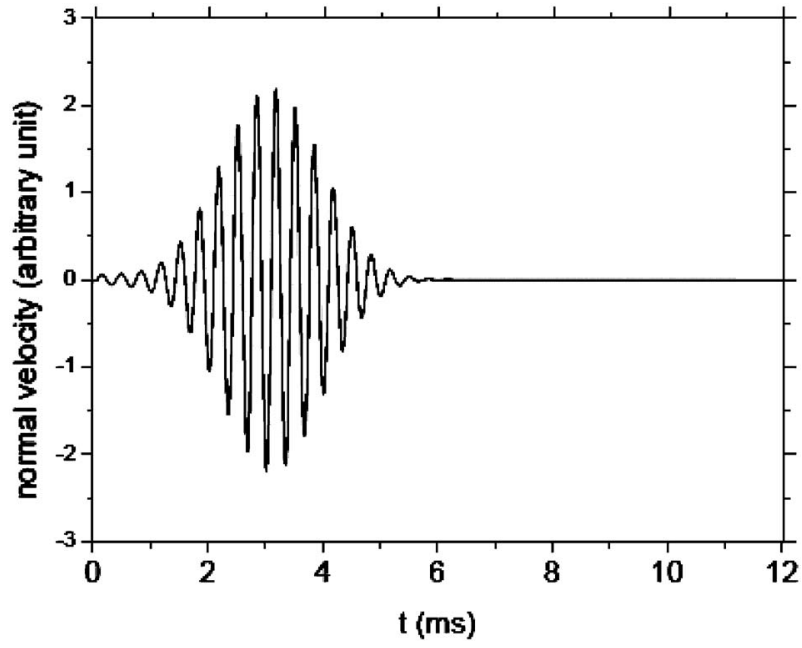

FIG. 7. Predicted normal velocity of the frame as a function of $t$, geometry a of Fig. 4, $r=3 \mathrm{~cm}$, semi-infinite layer.

contribution with the signal at the closest distance. The phase velocity is determined from the slope of the arrival times as a function of distance. These phase velocities are given in Table III. The variations from 2 to $4 \mathrm{kHz}$ are of the same order of magnitude as the precision of the measurements, which is around $5 \%$ and the measured values at $2 \mathrm{kHz}$ have been used in the whole range of frequencies. It has not been possible to evaluate the damping in a consistent way. The evaluation of the rigidity parameters is performed with Eqs. (55)-(57), the difference with the predictions of the Rayleigh wave speed with the Biot theory being smaller than the experimental imprecision. For the sake of simplicity, the loss angle of 0.1 of the simulation, slightly larger than the ones in Ref. 2, has been used for all the rigidity constants. For the geometry (b) of Fig. 4, a wave speed $1 / \operatorname{Re} q_{p}$ equal to $79 \mathrm{~m} / \mathrm{s}$ is obtained for $N=260(1+0.1 j) \mathrm{kPa}$ with Eq. (56). For the geometry $(\mathrm{a})$, the couples $(C, L)$ related to a wave speed equal to $51 \mathrm{~m} / \mathrm{s}$ are obtained with Eq. (55). In Fig. 9, $\operatorname{Re} L$ is represented as a function of $\operatorname{Re} C$. For these couples, Eq. (57) is used to evaluate the wave speed for the geometry (c) of Fig. 4. The phase velocity is represented in Fig. 10 as a function of $\operatorname{Re} C$. The wave speed of $36 \mathrm{~m} / \mathrm{s}$ is obtained for the couple $C=46(1+0.1 j) \mathrm{kPa}, L=125(1+0.1 j) \mathrm{kPa}$. For the full Biot theory the flow resistivities of Table I are the ones measured by Tarnow. ${ }^{2}$ The other acoustical parameters have not been measured but reasonable values have been chosen. For a similar medium, the evaluations by Tarnow ${ }^{2}$ are $\operatorname{Re} N=750 \mathrm{kPa}, \operatorname{Re} C=12 \mathrm{kPa}$, and $\operatorname{Re} L=40 \mathrm{kPa}$. The anisotropy is stronger in the measurements of Ref. 2 than in the present measurements but the same trend, a much larger rigidity in the directions perpendicular to the symmetry axis than in the direction of the axis, is observed.

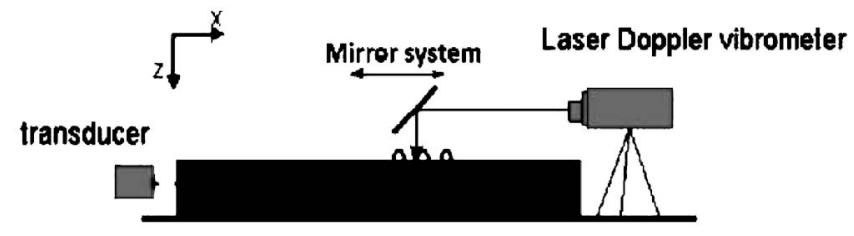

FIG. 8. Experimental setup.

TABLE III. Measured Rayleigh wave speeds for the different geometries represented in Fig. 4.

Wave speed $(\mathrm{m} / \mathrm{s})$ case (a)

Wave speed $(\mathrm{m} / \mathrm{s})$ case $(\mathrm{b})$
Frequency $(\mathrm{kHz})$

Wave speed $(\mathrm{m} / \mathrm{s})$ case $(\mathrm{c})$

\begin{tabular}{ccc}
2 & 3 & 4 \\
51 & 51 & 48 \\
79 & 78 & 80 \\
36 & 36 & 36 \\
\hline
\end{tabular}

\section{CONCLUSION}

A description of the Rayleigh waves that propagate in plane perpendicular or parallel to the symmetry axis of air saturated axisymmetrical soft porous layers is performed. This description is obtained from a generalization of previous works where the bulk waves are defined by the projection of the slowness vector in a plane perpendicular to the symmetry axis. The projected slowness vector is defined at any angle of the plane and the axis. Simulations show that some general properties of the Rayleigh waves on isotropic soft porous layers saturated by air can be observed for Rayleigh waves created on similar axisymmetrical media by a line source on free surfaces parallel and perpendicular to the symmetry axis in different directions. The wave speed of the Rayleigh wave for the frame in vacuo is not noticeably modified by air. The frame normal velocity is mainly due to the contribution of the Rayleigh wave, even at small distances from the line source. The finite thickness effect can be neglected for samples with a thickness of several centimeters in the medium frequency range. An illustration is given with an evaluation of the rigidity parameters of a glass wool from the measured Rayleigh wave speeds in three perpendicular directions. The frame in vacuo is strongly anisotropic. When the rigidity of the frame is an important parameter, for instance, when a porous layer is bonded to a metallic structure to increase the damping, this anisotropy should be taken into account.

\section{APPENDIX: THE COEFFICIENTS $\boldsymbol{A}_{l}$ AND $\boldsymbol{A}_{l}^{\prime}$}

$$
\begin{aligned}
& A_{0}=\bar{q}_{n}^{6} T_{0,6}+\bar{q}_{n}^{4} T_{0,4}+\bar{q}_{n}^{2} T_{0,2}+T_{0,0}, \\
& A_{1}=-2 \bar{q}_{x} \tan \varphi\left(3 \bar{q}_{n}^{4} T_{0,6}+2 \bar{q}_{n}^{2} T_{0,4}+T_{0,2}\right),
\end{aligned}
$$

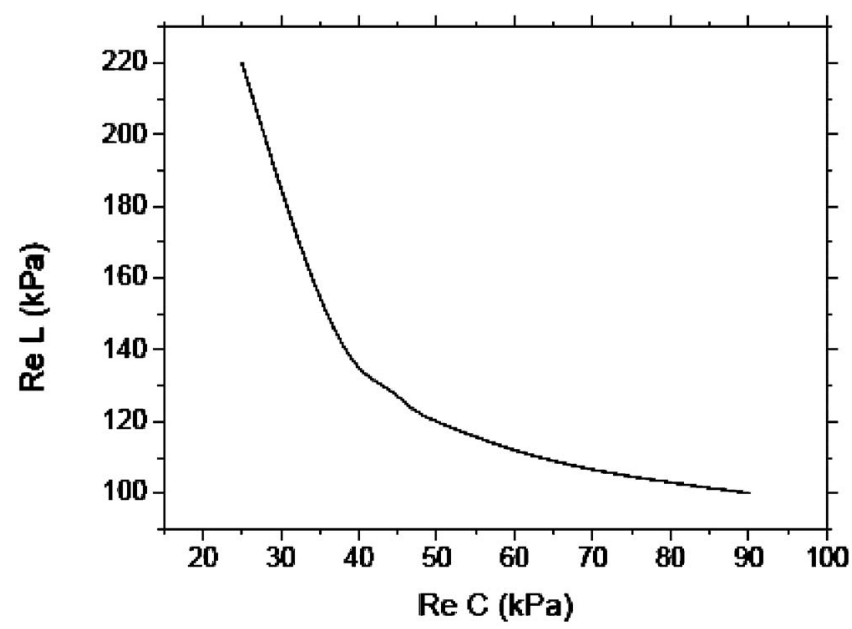

FIG. 9. $\operatorname{Re} L$ as a function of $\operatorname{Re} C$ for a wave speed of $51 \mathrm{~m} / \mathrm{s}$ of the Rayleigh wave in case (a), $\operatorname{Re} N=260 \mathrm{kPa}$. 


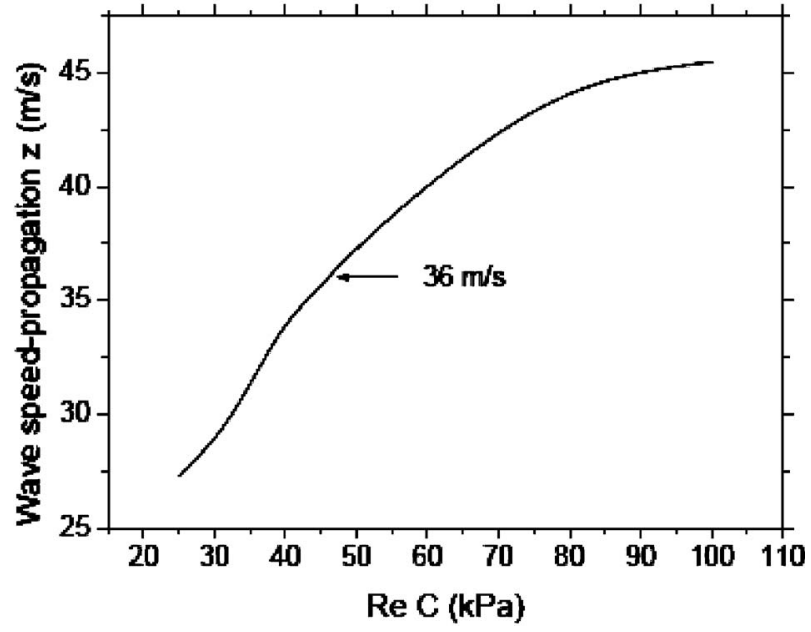

FIG. 10. Wave speed of the Rayleigh wave in case (c) as a function of $\operatorname{Re} C$, wave speed of the Rayleigh wave in case (a) $51 \mathrm{~m} / \mathrm{s}$, and $\operatorname{Re} N=260 \mathrm{kPa}$.

$$
\begin{aligned}
A_{2}= & \bar{q}_{n}^{4} T_{1,4}+\bar{q}_{n}^{2} T_{1,2}+T_{1,0}+\tan ^{2} \varphi\left(3 T _ { 0 , 6 } \left(q_{y}^{4}+6 \bar{q}_{x} q_{y}^{2}\right.\right. \\
& \left.\left.+5 \bar{q}_{x}^{4}\right)+2\left(q_{y}^{2}+3 \bar{q}_{x}^{2}\right) T_{0,4}+T_{0,2}\right), \\
A_{3}= & -2 \bar{q}_{x} \tan \varphi\left(2 \bar{q}_{n}^{2} T_{1,4}+T_{1,2}+\tan ^{2} \varphi\left[T_{0,6}\left(6 q_{y}^{2}+10 \bar{q}_{x}^{2}\right)\right.\right. \\
& \left.\left.+2 T_{0,4}\right]\right), \\
A_{4}= & \bar{q}_{n}^{2} T_{2,2}+T_{2,0}+\tan ^{2} \varphi\left[2 T_{1,4}\left(q_{y}^{2}+3 \bar{q}_{x}^{2}\right)+T_{1,2}\right] \\
& +\tan ^{4} \varphi\left[3 T_{0,6}\left(q_{y}^{2}+5 \bar{q}_{x}^{2}\right)+T_{0,4}\right], \\
A_{5}= & -2 \bar{q}_{x} \tan \varphi\left(T_{2,2}+2 \tan ^{2} \varphi T_{1,4}+3 \tan ^{4} \varphi T_{0,6}\right),
\end{aligned}
$$

$$
\begin{aligned}
A_{6}= & T_{3}+\tan ^{2} \varphi T_{2,2}+\tan ^{4} \varphi T_{1,4}+\tan ^{6} \varphi T_{0,6}, \\
A_{0}^{\prime}= & T_{0,0}^{\prime}+T_{0,2}^{\prime} \bar{q}_{n}^{2}, \quad A_{1}^{\prime}=-2 \bar{q}_{x} T_{0,2}^{\prime} \tan \varphi, \quad A_{2}^{\prime}=1 \\
& +T_{0,2}^{\prime} \tan ^{2} \varphi .
\end{aligned}
$$

${ }^{1}$ M. Melon, E. Mariez, C. Ayrault, and S. Sahraoui, J. Acoust. Soc. Am. 104, 2622 (1988).

${ }^{2}$ V. Tarnow, J. Acoust. Soc. Am. 118, 3672 (2005).

${ }^{3}$ M. D. Sharma and M. L. Gogna, J. Acoust. Soc. Am. 90, 1068 (1991).

${ }^{4}$ A. K. Vashishth and P. Khurana, J. Sound Vib. 277, 239 (2004).

${ }^{5}$ P. Khurana, L. Boeckx, W. Lauriks, P. Leclaire, O. Dazel, and J. F. Allard, J. Acoust. Soc. Am. 125, 915 (2009).

${ }^{6}$ O. Dazel, B. Brouard, C. Depollier, and S. Griffiths, J. Acoust. Soc. Am. 121, 3509 (2007).

T. Pritz, J. Sound Vib. 72, 317 (1980).

${ }^{8}$ T. Pritz, J. Sound Vib. 106, 161 (1986).

${ }^{9}$ H. J. Rice and P. Göransson, Int. J. Mech. Sci. 41, 561 (1999).

${ }^{10}$ K. U. Ingard, Notes on Sound Absorption Technology (Noise Control Foundation, Poughkeepsie, NY, 1994).

${ }^{11}$ R. Wilson and A. Cummings, Proceedings of the 1996 International Congress on Noise Control Engineering, Liverpool, UK (unpublished), pp. 269-272.

${ }^{12}$ S. Feng and D. L. Johnson, J. Acoust. Soc. Am. 74, 906 (1983).

${ }^{13}$ J. F. Allard, G. Jansens, G. Vermeir, and W. Lauriks, J. Acoust. Soc. Am. 111, 690 (2002)

${ }^{14}$ M. A. Biot, J. Appl. Phys. 33, 1482 (1962).

${ }^{15}$ J. F. Allard, Propagation of Sound in Porous Media, Modelling Sound Absorbing Materials (Elsevier, London, 1993).

${ }^{16}$ D. Royer and E. Dieulesaint, Ondes Elastiques Dans les Solides. I. Propagation Libre et Guidée (Masson, Paris, 1996).

${ }^{17}$ D. Lafarge, P. Lemarinier, J. F. Allard, and V. Tarnow, J. Acoust. Soc. Am. 102, 1995 (1997).

${ }^{18}$ D. L. Johnson, J. Koplik, and R. Dashen, J. Fluid Mech. 176, 379 (1987).

${ }^{19}$ N. Geebelen, L. Boeckx, G. Vermeir, W. Lauriks, J. F. Allard, and O. Dazel, J. Acoust. Soc. Am. 123, 1241 (2008). 